\title{
The physician at risk: disruptive behaviour, burnout, addiction, and suicide
}

\author{
Hilary P. Grocott, MD, FRCPC, FASE • Gregory L. Bryson, MD, FRCPC, MSc
}

Received: 9 November 2016/Accepted: 17 November 2016/Published online: 7 December 2016

(C) Canadian Anesthesiologists' Society 2016

\section{Physician, heal thyself}

Luke 4:23

The Journal has published an annual special theme issue in February of each year since 2011. Topics for these issues are carefully chosen after thoughtful discussions with the Journal editorial board and are informed by discussions with colleagues throughout the country and beyond. This year's compilation revolves around the complex and often interrelated issues of disruptive behaviour, burnout, addiction, and suicide. Although these are arguably somewhat sombre topics, we view them as necessary to begin conversations that will build and maintain the resilience, health, and professionalism of our specialty. This cluster of topics represents a departure from our usual theme issues whose content addresses more quantitative, scientific, and clinical matter. Indeed, our past two theme issues on Enhanced Recovery After Surgery ${ }^{1}$ and patientcentred outcome measures ${ }^{2}$ have focused firmly on patient experience.

Increasingly, however, attention has been directed to the health - both physical and mental - of the healthcare provider. Accordingly, the adage, "Physician, heal thyself", first put forth as a biblical proverb, raises the issue that, in caring for our patients, we often overlook our

H. P. Grocott, MD, FRCPC, FASE ( $\square)$

Departments of Anesthesia \& Perioperative Medicine,

St. Boniface Hospital, University of Manitoba, CR3008 - 369

Tache Avenue, Winnipeg, MB R2H 2A6, Canada

e-mail: hgrocott@sbgh.mb.ca

G. L. Bryson, MD, FRCPC, MSc

Department of Anesthesiology and Pain Medicine, Ottawa Hospital Research Institute, University of Ottawa, Ottawa, ON, Canada own health and well-being. If we are to care for our patients expertly and confidently, we must first ensure our own health.

For those who doubt the importance of this topic, we need only look at rates of suicide among our colleagues. Most of us do not have far to look back and recall a colleague who, unforeseen to those around them, ended up the subject of purposeful or accidental self-harm in the form of addiction or suicide. Understanding issues of workrelated stress, burnout, and other related mental health disorders among our colleagues is literally a matter of life and death.

Some readers may consider certain of these topics as somewhat taboo, perhaps because of the perception that they are not as organic as other non-mental health-related illnesses. Nevertheless, we need only look to the article on the biology of addiction ${ }^{3}$ to see just how science continues to elaborate on the complex etiology and pathophysiology of addiction-related brain disorders.

The interconnectedness of many of the topics in this special issue is particularly interesting. Although it may be easy to imagine burnout leading to addiction or suicide, the article by Yang $^{4}$ on professionalism raises other less intuitive connections. The concept of professionalism has ethical behaviour at its core. Disruptive behaviour, outlined twice in this issue, ${ }^{5,6}$ can result when professionalism is abrogated. Furthermore, burnout and other emotional exhaustion, as Dr. Yang highlights, can also increase our chances of callous (i.e., unprofessional) dealings toward patients. Though physician wellness is perhaps a less wellrecognized aspect of professionalism, it is nonetheless an important constituent. For example, if we ourselves are not well, we cannot serve our patients well. If we fail in maintaining our own fitness to practice, we fail in our societal contract as physicians. 
One notable article in this collection is a letter by Villafranca et al. ${ }^{6}$ reporting the results of a broad survey of healthcare workers concerning disruptive behaviour observed in the operating room. The letter highlights reports of "negative intraoperative behaviours" that include actual physical and psychological abuse in the operating room! Even though the denominator in the Villafranca survey is uncertain, the report of hundreds of abuse cases in Canadian operating rooms in a single year is nothing short of shocking. Clearly this represents unprofessional behaviour at its most basic level. Those who doubt the veracity of such a report need only look within their own institution - many of us could easily see similar conduct that likely represents the early roots of potentially negative behaviour. The tacit and arguably pervasive acceptance of even the most mild forms of disruptive behaviour is a slippery slope that likely contributed to some of the more egregious events reported.

Despite the admittedly dark tone of this Journal's special issue, there are also some bright spots. For example, Drs Kuhn and Flanagan offer tangible steps to improved wellness and increased resilience. ${ }^{7}$ Dr. Chakravarti et al. from the University of Saskatchewan bring us another hopeful message. ${ }^{8,9}$ They outline programmatic changes that can be made very early in the training of anesthesiologists. Their approaches represent specific preventative strategies that, if instituted early in the anesthesia training process, could provide anesthesia departments with the tools to prevent and treat the overly stressed and "at risk" anesthesiologist.

Our themed issues have always centred on moving the science of anesthesia forward with the ultimate goal of optimizing the care of patients. This compilation of articles now extends this concept. It highlights evidence that identifies physicians as an "at risk" population, and it offers strategies to enhance wellness in our anesthesia community. By doing so, we can only further enhance the care of our patients - let the healing begin.

\section{Médecins à risque: comportements perturbateurs, épuisement professionnel, dépendance et suicide}

Médecin, guéris-toi

Luc 4:23

Depuis 2011, le Journal publie chaque année un numéro spécial en février. Les sujets de ces numéros sont choisis avec soin après maintes discussions réfléchies au sein du comité de rédaction du Journal, et sont éclairées par des discussions avec nos collègues de partout au pays et d'ailleurs. La compilation de cette année a pour point focal les questions complexes et souvent imbriquées qui touchent aux comportements perturbateurs, à l'épuisement professionnel, à la dépendance et au suicide. Bien que ces sujets soient quelque peu funestes, nous croyons qu'ils sont néanmoins nécessaires si l'on souhaite lancer les conversations qui permettront de façonner et de maintenir la résilience, la santé et le professionnalisme de notre spécialité. Cet ensemble de thèmes constitue un départ net de nos numéros spéciaux habituels, dont le contenu est souvent de nature plus quantitative, scientifique et clinique. En effet, nos deux derniers numéros spéciaux portaient sur le rétablissement amélioré du patient après une chirurgie $^{1}$ et sur les mesures d'aboutissement centrées sur le patient ${ }^{2}$; leur propos se concentrait donc clairement sur l'expérience du patient.

Cependant, l'attention se porte de plus en plus sur la santé, tant physique que mentale, du professionnel de la santé. Dès lors, l'adage «médecin, guéris-toi », apparu d'abord sous forme de proverbe biblique, met en évidence le problème suivant: lorsque nous prenons soin de nos patients, nous mettons souvent de côté notre propre santé et notre bien-être personnel. Or, si nous souhaitons nous occuper de nos patients d'une main experte et assurée, nous devons en premier lieu garantir notre propre bonne santé.

Pour ceux qui douteraient de la pertinence de ce sujet, il suffit d'observer les taux de suicide parmi nos collègues. La plupart d'entre nous n'ont pas à chercher longtemps leur mémoire avant d'y trouver le souvenir d'un collègue qui, sans éveiller le moindre soupçon dans son entourage, a coupé court à la conversation en se faisant du mal, volontairement ou accidentellement, sous forme de dépendance ou de suicide. Il est absolument crucial de comprendre les problèmes de stress professionnel, d'épuisement professionnel et les autres troubles de santé mentale associés parmi nos collègues: c'est là une question de vie ou de mort.

Bien que certains des sujets abordés ici pourraient être considérés comme tabous, peut-être en raison de la perception selon laquelle ils ne sont pas aussi 'organiques' ou naturels que d'autres maladies non liées à la santé mentale, il suffit de consulter l'article sur la biologie de la dépendance ${ }^{3}$ pour voir comment la science n'a de cesse d'explorer plus avant l'étiologie complexe et la physiopathologie des troubles mentaux liés à la dépendance.

L'interconnectivité de bon nombre des sujets présentés dans ce numéro spécial est fascinante. Bien qu'on puisse facilement imaginer qu'un épuisement professionnel entraîne une dépendance ou un suicide, l'article de Yang ${ }^{4}$ 
concernant le professionnalisme soulève d'autres connexions encore, moins intuitives. Le comportement éthique constitue le cœur même du concept de professionnalisme. Les comportements perturbateurs, soulignés à deux reprises dans ce numéro, ${ }^{5,6}$ peuvent survenir lorsqu' on fait fi du professionnalisme. En outre, comme le souligne Dr Yang, l'épuisement professionnel, comme toute autre forme d'épuisement émotionnel, peut également augmenter nos chances d'interactions insensibles (c'est-à-dire de manque de professionnalisme) avec nos patients. Bien qu'il s'agisse peut-être d'un élément moins reconnu du professionnalisme, le bien-être du médecin n'en est pas moins une composante importante. Par exemple, si nous ne sommes pas en bonne santé nous-mêmes, nous ne pouvons bien servir nos patients. $\mathrm{Si}$ nous ne maintenons pas notre propre aptitude à pratiquer, nous manquons à notre contrat sociétal de médecins.

La lettre de Villafranca et coll. ${ }^{6}$ constitue un article remarquable de cette collection et rapporte les résultats d'un vaste questionnaire réalisé auprès des travailleurs des soins de santé concernant les comportements perturbateurs observés en salle d'opération. Cette lettre décrit des comptes rendus de «comportements peropératoires négatifs », notamment d'abus physiques ou psychologiques concrets en salle d'opération! Bien que le dénominateur du questionnaire de Villafranca demeure incertain, ce compte rendu de centaines de cas d'abus dans les salles d'opération canadiennes au cours d'une seule année est tout simplement choquant. Il s'agit clairement de comportements non professionnels au niveau le plus fondamental. Que ceux qui remettent en question la véracité d'un tel compte rendu regardent simplement au sein de leur propre établissement : nous serions nombreux à être témoins de comportements semblables, qui représentent probablement les premiers pas de comportements négatifs potentiels. L'acceptation tacite et vraisemblablement largement répandue - des manifestations, même les moins 'graves', de comportements perturbateurs constitue une pente glissante qui a probablement contribué à certains des événements les plus extrêmes rapportés ici.

Malgré le ton sinistre assumé de ce numéro spécial du Journal, il comporte également des rais de lumière. Par exemple, les Drs Kuhn et Flanagan proposent plusieurs étapes concrètes pour améliorer le bien-être et augmenter la résilience des médecins. ${ }^{7}$ Un autre message d'espoir nous parvient du Dr Chakravarti et de ses collègues de l'Université de la Saskatchewan. ${ }^{8,9}$ Ces chercheurs décrivent des changements programmatiques qui peuvent être mis en œuvre dès le début de la formation des anesthésiologistes. Leurs approches constituent des stratégies préventives spécifiques qui, si elles sont mises en place au début du processus de formation en anesthésie, pourraient procurer aux départements d'anesthésie les outils pour prévenir et traiter l'anesthésiologiste trop stressé et « à risque ».

L'objectif de nos numéros spéciaux a toujours été de faire progresser la science de l'anesthésie, avec pour but ultime d'optimiser les soins de nos patients. Cette compilation d'articles étend désormais ce concept en mettant en exergue les données probantes décrivant les médecins comme population «à risque » et propose diverses stratégies pour améliorer le bien-être de notre communauté de l'anesthésie. Ce faisant, nous ne pouvons qu'améliorer encore les soins de nos patients - que la guérison commence.

Conflicts of interest None declared.

Editorial responsibility This submission was handled by Dr. Philip M. Jones, Associate Editor, Canadian Journal of Anesthesia.

\section{Conflit d'intérêt Aucun.}

Responsabilité éditoriale Cet article a été traité par Dr Philip M. Jones, rédacteur adjoint, Journal canadien d'anesthésie.

\section{References}

1. Kehlet $H$. Enhanced Recovery After Surgery (ERAS): good for now, but what about the future? Can J Anesth 2015; 62: 99-104.

2. Myles PS. Perioperative outcomes: are we asking the right questions? Can J Anesth 2016; 63: 138-41.

3. MacNicol B. The biology of addiction. Can J Anesth 2017; 64: this issue. DOI: 10.1007/s12630-016-0771-2

4. Yang H. Professionalism in anesthesia. Can J Anesth 2017; 64: this issue. DOI: $10.1007 / \mathrm{s} 12630-016-0738-3$

5. Villafranca A, Hamlin C, Enns S, Jacobsohn E. Disruptive behaviour in the perioperative setting: a contemporary review. Can J Anesth 2017; 64: this issue. DOI: 10.1007/s12630-0160784-x

6. Villafranca A, Hamlin C, Jacobsohn E; Intraoperative Behaviours Research Group. Physical and psychological abuse in Canadian operating rooms. Can J Anesth 2017; 64: this issue. DOI: 10.1007/ s12630-016-0777-9

7. Kunh CM, Flanagan EM. Self-care as a professional imperative: physician burnout, depression and suicide. Can J Anesth 2017; 64: this issue. DOI: $10.1007 / \mathrm{s} 12630-016-0781-0$

8. Chakravarti A, Raazi M, O'Brien J, Balaton B. Anesthesiology resident wellness program at the University of Saskatchewan: concept and development. Can J Anesth 2017; 64: this issue. DOI: 10.1007/s12630-016-0772-1

9. Chakravarti A, Raazi M, O'Brien J, Balaton B. Anesthesiology resident wellness program at the University of Saskatchewan: curriculum content and delivery. Can J Anesth 2017; 64: this issue. DOI: $10.1007 / \mathrm{s} 12630-016-0773-0$ 